Full Length Article

\title{
Field location and player roles as constraints on emergent 1-vs-1 interpersonal patterns of play in football
}

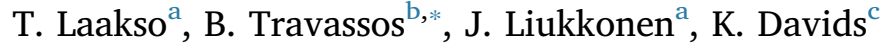 \\ ${ }^{\text {a } U n i v e r s i t y ~ o f ~ J y v a ̈ s k y l a ̈, ~ F i n l a n d ~}$ \\ ${ }^{\mathrm{b}}$ Research Centre for Sports Sciences, Health Sciences and Human Development (CIDESD), Universidade da Beira Interior, Covilhã, Portugal \\ ${ }^{\mathrm{c}}$ Centre for Sports Engineering Research, Sheffield Hallam University, UK
}

\section{A R T I C L E I N F O}

\section{Keywords:}

Performance analysis

Game constraints

Interpersonal coordination

Patterns of play

Decision-making

\begin{abstract}
A B S T R A C T
This study examined effects of player roles on interpersonal patterns of coordination that sustain decision-making in 1-vs-1 sub-phases of football in different field locations near the goal (left-, middle- and right zone). Participants were fifteen U-16 yrs players from a local competitive amateur team. To measure interpersonal patterns of coordination in the 1-vs-1 dyads we recorded: (i) the relative distance value between each attacker and defender to the centre of the goal, and (ii), the relative angle between the centre of the goal, each defender and attacker. Results revealed how variations in field locations near the goal (left-, middle- and right-zones) constrained the relative distance and relative angle values that emerged between them and the goal. It reveals that relative position of the goal is a key informational variable that sustained participants' behaviours for dribbling and shooting. Higher values of relative distance and angle were observed in the middle zone, compared to other zones. Players' roles also constitute a constraint on the interpersonal coordination for dribbling and shooting. Additionally, it seems that players' foot preference constrains the dynamics of interpersonal patterns of coordination between participants, especially in left and right zones. The findings suggest that to increase participants' opportunities for action, coaches should account with field positions, players' roles and preference foot.
\end{abstract}

\section{Introduction}

In the past decade researchers have increasingly recognized decision-making in team sports as one of the most influential aspects explaining performance (Araújo, Davids, \& Hristovski, 2006; Griffin \& Butler, 2005; Gréhaigne, Bouthier, \& David, 1997; Turner \& Martinek, 1995). Decision-making in team sports has been previously investigated with the aims of describing and explaining emergent behaviours of participants from an ecological dynamics perspective. Accordingly, decision-making emerges from a coupling of perception and action, predicated on individuals' action capabilities and information in a performance environment for identifying action possibilities (i.e., affordances) in line with specific intentions and task goals (Araújo et al., 2006; Fajen, Riley, \& Turvey, 2009; Paterson, Van der Kamp, Bressan, \& Savelsbergh, 2016).

This perspective proposes that decision-making should be investigated through identification of information that sustains individual behaviours and changes in emergent coordination tendencies between participants and teams (Araújo, Davids, Chow, \& Passos, 2009; Passos, Araújo, Davids, \& Shuttleworth, 2008). To achieve that aim, interactions between performers and their surroundings have been studied through identifying spatiotemporal patterns of interpersonal coordination that sustain actions in

\footnotetext{
* Corresponding author at: Universidade da Beira Interior, Departamento de Ciências do Desporto, Convento de Sto António, 6201-001 Covilhã, Portugal.

E-mail address: bruno.travassos@ubi.pt (B. Travassos).
} 
specific competitive performance contexts (Bartlett, Button, Robins, Dutt-Mazumder, \& Kennedy, 2012; Castellano \& Álvarez, 2013; Duarte et al., 2012; Sampaio, Lago, Gonçalves, Maçãs, \& Leite, 2013; Travassos, Araújo, Vilar, \& McGarry, 2011). In this line of reasoning, attacker-defender couplings have been deemed the fundamental unit of analysis for studying spatiotemporal relations that emerge between competing performers in team game performance (Davids, Araújo, \& Shuttleworth, 2005; McGarry, Anderson, Wallace, Hughes, \& Franks, 2002).

Previous research has sought to develop understanding of the forged and broken couplings that continuously emerge in attackerdefender dyadic systems. For instance, in basketball, interpersonal distance was identified as a key physical variable for explaining interpersonal interactions in a competitive dyadic system (Araújo et al., 2006). Following such ideas, it was observed in rugby union that interpersonal distance values of less than $4 \mathrm{~m}$, combined with relative velocity of at least $1 \mathrm{~m} / \mathrm{s}$, was influential in predicting an attacker running past the defender with the ball in 1-vs-1 dyads (Passos, Araújo, Davids, Gouveia, et al., 2008). In football, the values of interpersonal distance and relative velocity, capturing interpersonal relations in such dyads have revealed some contextual dependency, based on proximity-to-goal. Previous research has revealed that changes in proximity-to-goal of 1 -vs-1 (near to far from the goal) dyads influenced decision-making behaviours and intentionality of participants in relation to the ball (Headrick et al., 2011). In analyses of performance in 5-a-side futsal games it has also been reported that the angle to the goal is a key informational variable that sustained performers' behaviours in shooting at goal (Travassos et al., 2011; Vilar et al., 2012). The relevance of this interpersonal relation needs to be considered to understand decision-making behaviours in 1 -vs-1 football dyads (Clemente, Couceiro, Martins, Dias, \& Mendes, 2013)

Based in the extant literature further work is needed to consider variations in performance contexts and to provide information to impact significantly on coaching practice (Mackenzie \& Cushion, 2012). There is also a need to understand how interpersonal patterns of coordination between attackers and defenders in 1-vs-1 dyads are influenced by field location effects relative to the goal. The specific aim of this study was to analyse patterns of interpersonal coordination that sustain decision-making of performers in 1-vs-1 sub-phases of football in different field locations near the goal (in left-, middle- and right- zones of the attacking third on field). Based on previous work, we expected to observe an effect of field location on emergent patterns of coordination in 1-vs-1 sub-phases. Furthermore, we also investigated effects of players' roles (e.g., attackers, midfielders and defenders) on interpersonal patterns of coordination that underpin decision-making in 1-vs-1 sub-phases in football. Based on previous research (Gonçalves, Figueira, Maçãs, \& Sampaio, 2014), suggesting that different technical and tactical abilities of players with different roles support their exploration of interpersonal relations with opponents, we expected to observe different patterns of coordination emerging, depending on participants' main roles as defenders or attackers.

\section{Methods}

\subsection{Participants}

Fifteen male players (under-15 yrs age group; mean age $13.2 \pm 1.03$ years; years of practice $4.2 \pm 1.10$ years) participated in this study, categorised according to their team role, resulting in 5 defenders, 7 midfielders and 3 attackers. All players were rightfooted and played in the club's first team. Players typically undertook four field training sessions per week ( $\sim 90$ min per session) plus a gym session ( $\sim 60$ min per session) to improve balance, coordination and strength, and played a competitive game at the weekend. The club and parents of participants provided prior informed consent for participation in the study. The study was approved and accepted by the Ethics local Committee according to the Declaration of Helsinki.

\subsection{Task and procedures}

Each participant was asked to perform in the role of a ball dribbler (attacker) and defender at three field locations. Attackerdefender dyads competed in an area of $10 \mathrm{~m} \times 5 \mathrm{~m}$ positioned to represent the different locations (described below) under competitive performance conditions. The starting distance between attacker and defender was $3 \mathrm{~m}$ (see Fig. 1). At the end of this area, there was the goalkeeper's area. A regular size football goal $(2.44 \mathrm{~m} \times 7.32 \mathrm{~m})$ protected by a goalkeeper was used. Participants were divided in three groups according their playing position on the field (defender, midfielder or attacker). All participants performed in the 1-vs-1 trials starting from all three zones as an attacker and also as a defender, resulting in a total number of 129 trials. In order to seek reliability of the tracking system, a sequential order to the roles participants were required to adopt between field zones. All trials were initiated first from the right zone, then from the midfield zone and last from the left zone. To ensure that participants sought to constantly use adaptability during the emerging interactions between attackers and defenders, dyadic system opponents were changed trial by trial (i.e., participants intermittently switched between acting as attackers and as defenders from trial to trial). All the participants had time to rest between trials in order to avoid fatigue effects. In order to ensure a balanced number of trials per player role, each defender performed three trials, each midfielder performed two trials and each attacker performed six trials in each field zone.

Each trial started when both the attacking and defending participants were ready in their starting positions and the attacking player was requested to start the trial. As soon as attacker moved the ball, the defender was allowed to start defending. The aim of the attacker was to dribble past the defender and shoot at goal. If this occurred, the trial was over. The aim of the defender was to prevent the attacker from scoring a goal, within the laws of the game. The trial was considered completed when the ball moved outside the borders of the playing area (A regulation ball size 5 was used in all trials). All the trials that ended with a shot at goal or with the ball moved outside the borders of the playing area, without the ball carrier dribbling past the defender, were removed from further 


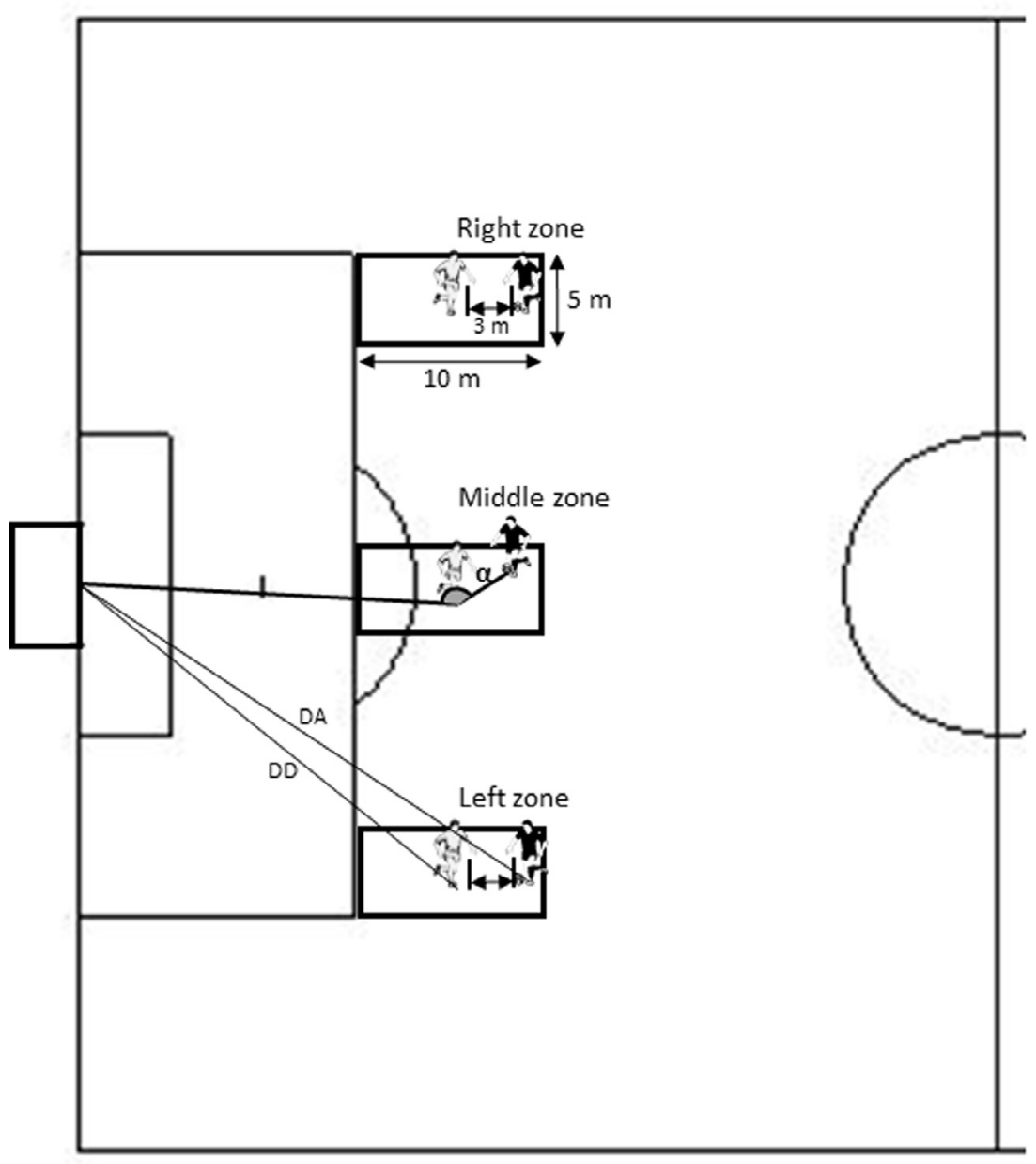

Fig. 1. Representation of the three areas of play (left, middle, right) with the definition of the starting zone and their location in relation to the goal. $\alpha$ - represents the relative angle between goal, defender and attacker player. DD - represents the distance between defender to the centre of goal. DA - represents the distance between attackers to the centre of goal.

analysis in the study. The elimination of such trials helped us to only capture and describe the interactional dynamics during performance sequences when the ball carrier successfully dribbled past the defender. Twelve trials in total were removed from further analysis for this reason.

Each participant's movements were captured by using a digital video camera (Sony HRX-MC50E) placed $4 \mathrm{~m}$ above ground forming an angle of approximately $45^{\circ}$ with the longitudinal axis of the performance area to capture movements during the whole task. All the video recordings captured the displacement trajectories of all participants without moving the camera. The video recordings were digitized with TACTO software (see, Duarte et al., 2010, for additional information). The displacement trajectories of the ball and participants were tracked using a computer mouse, by following, in every frame, the projection of their centre of gravity on the playing surface. The obtained coordinates were transformed into real coordinates using the direct linear transformation method (2D-DLT) and filtered with a Butterworth low pass filter (6 Hz) (Winter, 2005).

\subsection{Reliability}

Ten trials were selected at random and the displacement trajectories of attacker and defender players $(n=20)$ were re-digitised by the same experimenter. Intra-digitiser reliability were assessed using technical error of measurement (TEM) and coefficient of reliability (R) (N.B. TEM $=\Sigma D^{2} / 2 N$, where $D$ is the difference between pre- and post-test measures and $N$ is the sample size. $R=1-\mathrm{TEM}^{2} / S D^{2}$, where $S D$ is the standard deviation of all measures) (Goto \& Mascie-Taylor, 2007). The intra-TEM yielded values of $0.254 \mathrm{~m}(2.43 \%)$ with a corresponding coefficient of reliability $(R=0.981)$.

\subsection{Data analysis}

To measure variations in interpersonal patterns of coordination between participants in the 1 -vs- 1 sub-phases, variations in 
relative distance between the attacker and defender players to the centre of goal (RDPG), and the relative angle ( $\alpha$ ) between the centre of goal, defender and attacker (RAGDA) (see Fig. 1), were calculated, based on methods used in previous research by Vilar et al. (2012). Values of RDPG were calculated as the difference between the value of the attacker's distance to the centre of the goal (DA) and the defender's distance to the centre of the goal (DD). Values of RAGDA were calculated by measuring the inner product of the defender's vector to the centre of the goal, and the defender's vector to the attacker (see Fig. 1). Due to differences in the temporal length of each trial, and for purposes of comparison, each trial was normalized to the total time taken to perform the trial independently. Data were averaged for every $10 \%$ portion of the total normalized time in each trial. The value of $0 \%$ corresponds to the moment of trial initiation (when the attacker was given a signal to start the trial with a dribble). The value of $100 \%$ corresponded to the moment when the attacker moved into the target zone to shoot at goal or when ball was played out of the performance area.

Magnitude-based inferences and precision of estimation were used to avoid the shortcomings of research approaches supported by null-hypothesis significance testing (Batterham \& Hopkins, 2006). Comparisons of RDPG and RAGDA data among field zones and players' roles were assessed via standardized mean differences, computed with pooled variance and respective $90 \%$ confidence intervals (Cumming, 2012; Hopkins, Marshall, Batterham, \& Hanin, 2009). The field zones comprised the left, middle and right performance areas, and the players' roles comprised the different combinations of Defenders, Midfielders and Attackers, functioning as attackers or defenders respectively (AADD - Attacker attacks, Defender defends (27 trials); AAMD - Attacker attacks, Midfielder defends (27 trials); DAAD - Defender attacks, Attacker defends (21 trials); DAMD - Defender attacks, Midfielder defends (18 trials); MAAD - Midfielder attacks, Attacker defends (18 trials); MADD - Midfielder attacks, Defender defends (18 trials)). Thresholds for effect sizes statistics were trivial (0-0.19); small (0.2-0.59); moderate (0.6-1.19); large (1.2-1.99); and very large ( $\geq 2.0$ ) (direction of observed effects were represented by -ive and +ive). Differences in means for both pairs of scenarios were also expressed in percentage units with 90\% confidence intervals (CI) (Hopkins et al., 2009). The relationships between values of relative distance and relative angles were analysed using Pearson's Product Moment Correlation using SPSS 22.0 software (IBM SPSS Inc., Chicago, USA).

\section{Results}

\subsection{The effects of field zones}

Analysis of relative distance values between players and the goal revealed main effects for field zones: Left-Middle $(d=-1.22$ (90\%CI: -1.62 to -0.83$)$, moderate - ive), Left-Right $(d=-0.75$ (90\%CI: -1.13 to -0.37 ), small - ive), and Right-Middle ( $d=0.49$ (90\%CI: 0.11-0.87), trivial). Generally, the left zone showed lower relative distance values between players and the goal than the other two zones, with the middle zone revealing the higher values. In the left zone, the relative distance decrease from values around 5-1.3 m. In the middle and right zones, relative distance started at values near $5.5 \mathrm{~m}$ and decreased in the middle to values around $2.5 \mathrm{~m}$ and on the right to values near $1.7 \mathrm{~m}$ (see Fig. 2, left panel).

Analysis of values of the relative angle between goal, defender and attacker revealed main effects for field zones: Left-Middle ( $d=-6.12$ (90\%CI: -6.98 to -5.25 ), very large - ive), Right-Middle ( $d=-5.67$ (90\%CI: 4.84-6.51), very large - ive, and LeftRight ( $d=-0.04$ (90\%CI: $-0.4-0.33)$, unclear). Generally, higher values of relative angle were observed in the middle zone, than in the left or right zones. In the middle zone angle values were near $180^{\circ}$ and in the left and right zones angle values were near $130^{\circ}-140^{\circ}$. Interestingly, at the end of the trial in the left zone, an increase in relative angle values to nearer $150^{\circ}$ was observed. In the right zone, relative angle values were maintained nearer to $135^{\circ}$ (see Fig. 2, right panel).

Analysis of relationships between values of relative distance and relative angle for each field zone revealed interesting effects. There was a strong negative correlation between the two variables in the left $(\mathrm{r}=-0.935, p<0.001)$ and right zone ( $\mathrm{r}=-0.992$, $p<0.001)$ and a strong positive correlation in the middle zone $(\mathrm{r}=0.963, p<0.001)$.

\subsection{The effects of player roles}

Analysis of relative distance values between players and the goal showed small effects for differences in player roles between DAAD-AADD ( $d=-0.6$ (90\%CI: -0.08 to -1.09 ), small - ive), DAAD-AAMD ( $d=-0.99$ (90\%CI: -0.47 to -1.53$)$, small - ive),
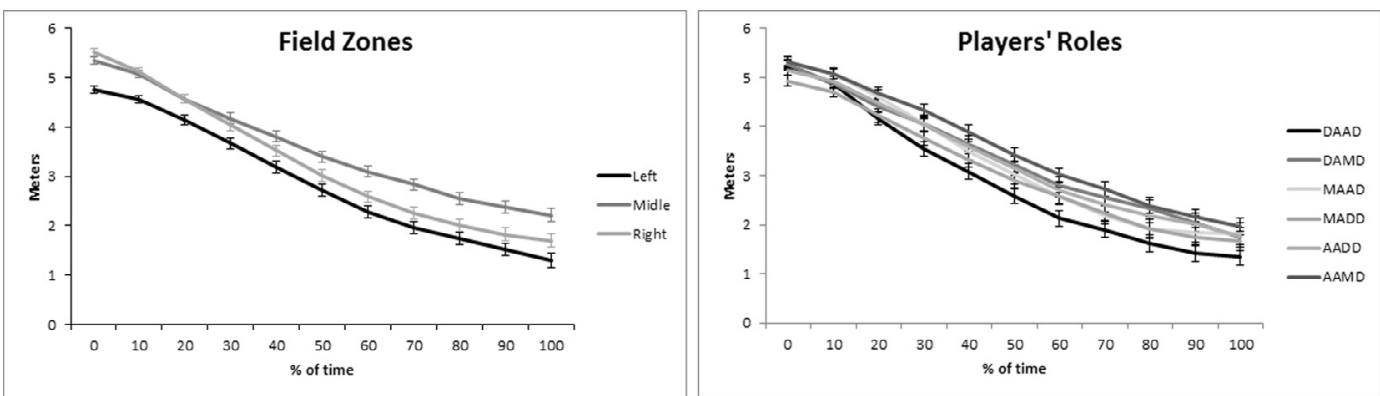

Fig. 2. Mean values and standard deviations of relative distance between attacker and defender to the centre of goal. Left panel - variations on mean relative distance according to field zones. Right panel - variations on mean relative distance according to players' roles. 

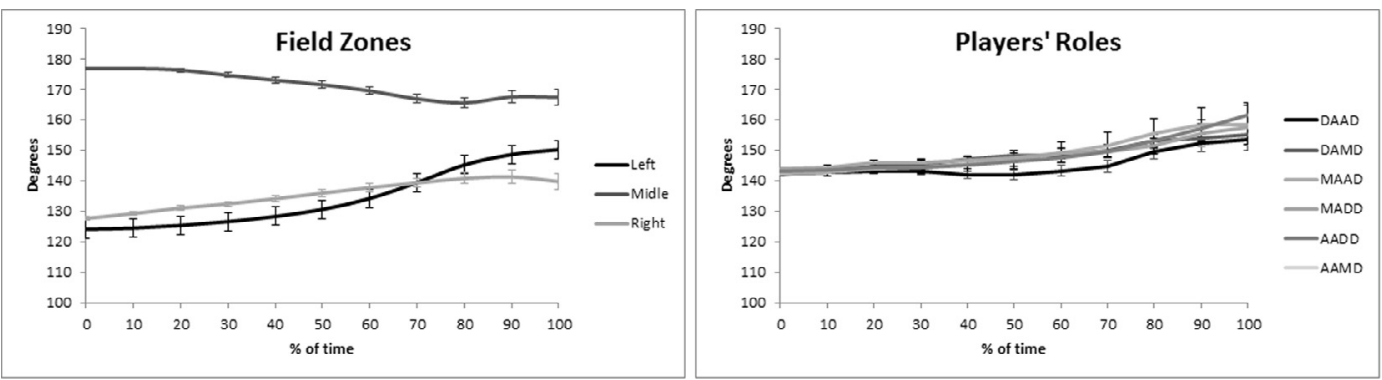

Fig. 3. Mean values and standard deviations of relative angle between goal, defender and attacker player. Left panel - variations on mean relative angle according to field zones. Right panel - variations on mean relative angle according to players' roles.

and DAAD-DAMD ( $d=-0.74$ (90\%CI: -1.29 to -0.18$)$, small - ive), DAAD-MAAD $(d=-0.6$ (90\%CI: -1.17 to -0.02$)$, small -ive). In general, patterns of play of defenders as attackers and attackers as defenders, compared to other roles, revealed lower values of relative distance at the end of the trials (see Fig. 3, left panel).

Analysis of relative angle between goal, defender and attacker player revealed unclear effects of player role (see Fig. 3, right panel).

Analysis of relationships between values of relative distance and relative angle for each dyad revealed a strong negative correlation between the two variables, AADD ( $\mathrm{r}=-0.860, p<0.001)$; AAMD $(\mathrm{r}=-0.866, p<0.001)$; DAA ( $\mathrm{r}=-0.697$, $p<0.05)$; DAMD ( $\mathrm{r}=-0.975, p<0.001)$; MAAD $(\mathrm{r}=-0.915, p<0.001)$; MADD $(\mathrm{r}=-0.899, p<0.001)$. Interestingly, the weakest correlations were observed between defenders as attackers and attackers as defenders, in line with previous research findings.

\section{Discussion}

In this study, we sought to examine the interpersonal patterns of coordination that sustained decision-making of participants in 1vs1 sub-phases in football at different field locations near the goal (left-, middle- and right-zones). Also, the effect of players' roles (i.e., attackers, midfielders and defenders) on interpersonal patterns of coordination in 1-vs-1 sub-phases in football was analysed.

In line with previous research, the results clearly confirmed an effect of field locations on emergent interpersonal patterns of coordination between an attacker and defender in 1-vs-1 sub-phases (Headrick et al., 2011). Headrick et al. (2011) showed how proximity-to-goal constrained values of defender to ball distance. Our results revealed how variations in field locations near the goal (left-, middle- and right-zones) constrained interpersonal patterns of coordination between attackers and defenders, particularly the relative distance and relative angle values that emerged between them and the goal. In line with other previous studies, our results highlighted relative position of the goal as a key informational variable that sustained participants' behaviours for dribbling and shooting (Travassos et al., 2011; Vilar et al., 2012). Changes in the value of the informational variable 'angle to goal' constrained the dynamics of the 1vs1 dyad, with clear implications for the interpersonal relations that participants explored to be successful, namely the distances and angles between them. Additionally, the exploration of possibilities for action in the 1vs1 dyad was constrained by players' main roles according to the relative position on-field. It is likely that the participants' past experiences in a specific performance role may have strongly influenced their tendencies for engaging in interpersonal coordination with other participants under the constraints of competition.

\subsection{The effect of field locations}

Higher values of relative distance between attackers and defenders were observed in the middle zone, compared to other zones. At the same time, results of relative angle values between players and the goal were also higher (close to $180^{\circ}$ ) in the middle zone, than in the left and right zones (near $130^{\circ}$ to $140^{\circ}$ ). The relationship between both variables revealed a positive correlation for middle zone in contrast to right and left zones which revealed negative correlations. A possible explanation for such positive correlations, with higher, more stable values of relative distance near to $180^{\circ}$ and higher distance values in the middle zone might be related to the high number of opportunities for ball dribblers to explore opportunities for shooting at goal. These results are in line with data reported in previous work by Vilar et al. (2012), suggesting that shooting opportunities emerged by attackers promoting a misalignment in their co- positioning with defenders relative to the ball and the goal. In their study defenders sought to maintain 'attacker-defender-goal symmetry' by placing themselves between the goal and the immediate attacker, maintaining a functional distance to intercept the ball or block a possible shot. This was a challenging task in the middle-zone since the actions of the defenders were constrained by greater opportunities for attackers to exploit space and move left, right or through the middle creating an open angle to shoot at goal. Since attackers had more such affordances (opportunities for action) with the ball, defenders were constrained to be more conservative in positioning, typically by increasing the value of their relative distance with the attacker (Headrick et al., 2011). Interestingly, similar behaviours have been observed at a team level after manipulations of the number of goal targets in a practice task (e.g., 3 goals rather than 1 goal to shoot at). Increasing the number of goal targets available for attackers resulted in the defending teams retreating on field and increasing the distance between them and the attacking team (Travassos, Gonçalves, Marcelino, Monteiro, \& Sampaio, 
2014). Increasing the number of possibilities for action promotes co-adaptations of participants and teams to adopt more conservative interpersonal patterns of coordination, characterized by greater distance values and stability in the spatial equilibrium/symmetry between performers and the goal(s) location (Travassos et al., 2014).

We also observed lower values of relative distances in the left, compared to the right zone. Also, an increase in relative angles, at the end of the trial, to values near to $150^{\circ}$ was noted in the left zone. In the right zone, the relative angle variable maintained values near to $135^{\circ}$. Interestingly, negative correlations were observed between values of relative distances and angles. When the value of relative distance decreased, the result was an increase in the value of relative angle to maintain the alignment between players and the goal. Differences observed in the relative distance and relative angles, at the end of the trials, between participants in the left and right zones can be explained by the fact that all the players were right-footed. This physical characteristic meant that, in the left zone the attackers could attempt to dribble past the defender with the right foot to open up a shooting angle with the goal. In the right zone, dribbling with the right foot tended to close the shooting angle with the goal. Thus, in the left zone, to prevent attackers from using their favoured foot to dribble and open an angle for shooting at goal, defenders sought to minimize interpersonal distances and the relative angle to the goal. The observed increase in relative angle, at the end of the trial, in the left zone, may represent attempts of attackers to dribble, open the angle to goal and shoot with their favoured right foot. In line with the ecological approach and the notion of affordances, this finding suggests that the exploration of possibilities for action is forged on the relation between emergent spatial relations, relative to the capacities (effectivities) of participants to act and achieve specific performance aims (Araújo et al., 2006; Fajen et al., 2009; Paterson et al., 2016). Indeed, the interpersonal patterns of coordination observed were forged on the acquisition of a perception-action coupling between both attackers and defenders, considering their own action capabilities in relation to the determined spatial relations and the proposed task goals (Travassos et al., 2014; van Andel, Cole, \& Peping, 2017). Further research is required to better understand how variations in the specific capacities of sport performers (e.g., foot preference of participants, different levels of expertise, or even different physical capabilities and levels of fatigue) impact on the emergent dynamics of interpersonal patterns of coordination in different games sub-phases.

Clearly, implications for the design of practice tasks can be advocated. Attackers and defenders can be exposed to different relative positions to the goal for training dribbling and shooting, with changes in the preferred foot of both attackers and defenders. That personal constraint manipulation will encourage greater exploration of possibilities for action of attackers to shoot when presented with a more open or closed angle to the goal. Such a manipulation may even encourage participants to explore shooting with the non-preferred foot, depending on the affordances offered by information from the positioning of defenders, relative to the goal. Also, for defenders, such a manipulation will help them to improve their defensive positioning, relative to the goal, and also to identify and nullify use of the preferred foot of attackers. This exploration of capabilities for action of other performers, based on some key informational, will allow learners to become more effective and flexible in their behaviours (Button et al., 2013).

\subsection{The effect of players' roles}

Due to different technical and tactical abilities facilitating participants' exploration of the performance environment, it was also expected that different patterns of coordination would emerge in the 1-vs-1 sub-phase between participants with different roles in the squad (Gonçalves et al., 2014). Our results revealed that when a defender attacks, and an attacker defends, lower values in interpersonal distance emerged in comparison with other players' role combinations. This finding can be explained by the capability of opponents to perceive affordances (the potential for actions) of other people, as they can do for themselves (Mark, 2007), consequently changing the interpersonal patterns of coordination that sustain performance. Also, lower correlation values were observed between such variables in these player dyadic systems.

In fact, in competitive performance environments, defenders typically do not have many opportunities to experience1-vs-1 opportunities as attackers and vice versa, changing the exploration of the environment and potential for action when different roles are required (Travassos et al., 2013). Thus, the findings suggest that perception of the individual capabilities of the defenders to dribble and shoot at goal afforded a decrease in the relative distance between them as an option to reduce their possibilities for action (Travassos et al., 2012). Previous research (Vilar et al., 2012), has suggested that, when a ball dribbler was able to shoot and score a goal, he was able to maintain a significantly larger interpersonal distance value between him and a marking defender.

In line with an ecological dynamics approach, these findings suggested that participants' actions emerged from perception of information arising continuously from environmental interactions according to current capabilities for action of individuals (Araújo et al., 2006; Davids et al., 2005). Players' roles seem to have an impact on their current capabilities for action. Thus, to improve player performance, early experience of diverse experiences in the contexts of play and in required perception and action capacities instead of specialization (as defenders or attackers) should help learners to improve their adaptability to the different performance contexts to which they are exposed during competition (Davids, Araújo, Correia, \& Vilar, 2013).

\section{Conclusions}

To summarize, these data support the idea that different field locations near the goal (in left-, middle- and right- zone) constrain the interpersonal coordination that sustain 1-vs-1 sub-phases in football. Players' roles also constitute a constraint on the interpersonal coordination for dribbling and shooting. Data implied that players' foot preference can be considered a key constraint to define the action capabilities of attackers to explore the dribbling and shooting. The findings suggest that coaches should manipulate practice task constraints (i.e. design 1-vs-1 sub-phases in different locations on field and manipulating players' foot preferences on participants' dyads) to increase opportunities for the participants to become better attuned to the informational variables that 
constrain their performance. By manipulating task constraints, such as field location for attacker-defender dyads or individual constraints such as placing right- or left-footed participants in different areas of play, participants may learn how to detect functional information for decision-making in 1-vs-1 sub-phases.

\section{Funding}

This research was partly supported under Grants (FCT/UID/DTP/04045/2013) and COMPETE (POCI-01-0145-FEDER-006969) to CIDESD - Research Center in Sport, Health and Human Development.

\section{References}

Araújo, D., Davids, K., Chow, J. Y., \& Passos, P. (2009). The development of decision making skill in sport: An ecological dynamics perspective. In D. Araújo, H. Ripoll, \& M. Raab (Eds.), Perspectives on cognition and action in sport (pp. 157-170). New York: Nova Science Publishers.

Araújo, D., Davids, K., \& Hristovski, R. (2006). The ecological dynamics of decision making in sport. Psychology of Sport and Exercise, 7(6), 653-676. http://dx.doi.org/ 10.1016/j.psychsport.2006.07.002.

Bartlett, R., Button, C., Robins, M., Dutt-Mazumder, A., \& Kennedy, G. (2012). Analysing team coordination patterns from player movement trajectories in soccer: Methodological considerations. International Journal of Performance Analysis in Sport, 12(2), 398-424.

Batterham, A. M., \& Hopkins, W. G. (2006). Making meaningful inferences about magnitudes. International Journal of Sports Physiology and Performance, 1(1), 50-57.

Button, C., Chow, J., Travassos, B., Vilar, L., Duarte, R., Passos, P., ... Davids, K. (2013). A nonlinear pedagogy for sports teams as social neurobiological systema: How teams can harness self-organization tendencies. In A. Ovens, \& T. Hopper (Eds.), Complexity thinking in physical education (pp. 135-150). Oxon: Routledge.

Castellano, J., \& Álvarez, D. (2013). Defensive use of the interaction space in soccer. International Journal of Sport Science, 9(32), $126-136$.

Clemente, F. M., Couceiro, M., Martins, F., Dias, G., \& Mendes, R. (2013). Interpersonal dynamics: $1 \mathrm{v} 1$ sub-phase at sub-18 football players. Journal of Human Kinetics, 36(1), 179-189.

Cumming, G. (2012). Understanding the new statistics: Effect sizes, confidence intervals, and meta-analysis. New York: Routledge.

Davids, K., Araújo, D., Correia, V., \& Vilar, L. (2013). How small-sided and conditioned games enhance acquisition of movement and decision-making skills. Exercise and Sport Sciences Reviews, 41(3), 154-161.

Davids, K., Araújo, D., \& Shuttleworth, R. (2005). Applications of dynamical systems theory to football. In T. Reilly, J. Cabri, \& D. Araújo (Eds.), Science and football V: The proceedings of the fifth world congress on sports science and football (pp. 537-550). Routledge.

Duarte, R., Araújo, D., Davids, K., Travassos, B., Gazimba, V., \& Sampaio, J. (2012). Interpersonal coordination tendencies shape 1-vs-1 sub-phase performance outcomes in youth soccer. Journal of Sport Sciences, 30(9), 871-877. http://dx.doi.org/10.1080/02640414.2012.675081.

Duarte, R., Araújo, D., Fernandes, O., Fonseca, C., Correia, V., Gazimba, V., ... Lopes, J. (2010). Capturing complex human behaviors in representative sports contexts with a single camera. Medicina, 46(6), 408-414.

Fajen, B., Riley, M., \& Turvey, M. (2009). Information, affordances, and the control of action in sport. Internal Journal of Sport Psychology, 40(1), 79-107.

Gonçalves, B. V., Figueira, B. E., Maçãs, V., \& Sampaio, J. (2014). Effect of player position on movement behaviour, physical and physiological performances during an 11-a-side football game. Journal of Sports Sciences, 32(2), 191-199. http://dx.doi.org/10.1080/02640414.2013.816761.

Goto, R., \& Mascie-Taylor, C. G. N. (2007). Precision of measurement as a component of human variation. Journal of Physiological Anthropology, 26(2), 253-256. http:// dx.doi.org/10.2114/jpa2.26.253.

Gréhaigne, J. F., Bouthier, D., \& David, B. (1997). Dynamic-system analysis of opponent relationships in collective actions in soccer. Journal of Sports Sciences, 15(2), 137-149. http://dx.doi.org/10.1080/026404197367416.

Griffin, L. L., \& Butler, J. (2005). Teaching games for understanding: Theory, research, and practice. Human Kinetics.

Headrick, J., Davids, K., Renshaw, I., Araújo, D., Passos, P., \& Fernandes, O. (2011). Proximity-to-goal as a constraint on patterns of behaviour in attacker-defender dyads in team games. Journal of Sport Sciences, 30(3), 247-253. http://dx.doi.org/10.1080/02640414.2011.640706.

Hopkins, W., Marshall, S., Batterham, A., \& Hanin, J. (2009). Progressive statistics for studies in sports medicine and exercise science. Medicine \& Science in Sports \& Exercise, 41(1), 3-12.

Mackenzie, R., \& Cushion, C. (2012). Performance analysis in football: A critical review and implications for future research. Journal of Sports Sciences, 31(6), 639-676. http://dx.doi.org/10.1080/02640414.2012.746720.

Mark, L. S. (2007). Perceiving the actions of other people. Ecological Psychology, 19(2), 107-136.

McGarry, T., Anderson, D. I., Wallace, S. A., Hughes, M. D., \& Franks, I. M. (2002). Sport competition as a dynamical self-organizing system. Journal of Sports Sciences, 20(10), 771-781. http://dx.doi.org/10.1080/026404102320675620.

Passos, P., Araújo, D., Davids, K., Gouveia, L., Milho, J., \& Serpa, S. (2008). Information-governing dynamics of attacker-defender interactions in youth rugby union. Journal of Sports Sciences, 26(13), 1421-1429. http://dx.doi.org/10.1080/02640410802208986.

Passos, P., Araújo, D., Davids, K., \& Shuttleworth, R. (2008). Manipulating constraints to train decision making in Rugby Union. International Journal of Sports Science and Coaching, 3(1), 125-140.

Paterson, G., Van der Kamp, J., Bressan, E., \& Savelsbergh, G. (2016). Action-specific effects on perception are grounded in affordance perception: An examination of soccer players' action choices in a free-kick task. International Journal of Sport Psychology, 47(4), 318-334.

Sampaio, J., Lago, C., Gonçalves, B., Maçãs, V., \& Leite, N. (2013). Effects of pacing, status and unbalance in time motion variables, heart rate and tactical behaviour when playing 5-a-side football small-sided games. Journal of Science and Medicine in Sport, 17(2), $229-233$.

Travassos, B., Araújo, D., Davids, K., O'Hara, K., Leitão, J., \& Cortinhas, A. (2013). The effect of expertise on decision making in sport - A meta-analysis. Psychology of Sport \& Exercise, 14(2), 211-219. http://dx.doi.org/10.1016/j.psychsport.2012.11.002.

Travassos, B., Araújo, D., Davids, K., Vilar, L., Esteves, P., \& Correia, V. (2012). Informational constraints shape emergent functional behaviors during performance of interceptive actions in team sports. Psychology of Sport \& Exercise, 13(2), 216-223. http://dx.doi.org/10.1016/j.psychsport.2011.11.009.

Travassos, B., Araújo, D., Vilar, L., \& McGarry, T. (2011). Interpersonal coordination and ball dynamics in futsal (indoor football). Human Movement Science, 30, 1245-1259. http://dx.doi.org/10.1016/j.humov.2011.04.003.

Travassos, B., Gonçalves, B., Marcelino, R., Monteiro, R., \& Sampaio, J. (2014). How perceiving additional targets modifies teams' tactical behavior during football small-sided games. Human Movement Science, 38, 241-250.

Turner, A., \& Martinek, T. J. (1995). Teaching for understanding: A model for improving decision making during game play. Quest, 47(1), 44-63.

van Andel, S., Cole, M., \& Peping, G. J. (2017). A systematic review on perceptual-motor calibration to changes in action capabilities. Human Movement Science, 51, 59-71.

Vilar, L., Araújo, D., Davids, K., Travassos, B., Duarte, R., \& Parreira, J. (2012). Interpersonal coordination tendencies supporting the creation/prevention of goal scoring opportunities in futsal. European Journal of Sport Sciences, 14(1), 28-35. http://dx.doi.org/10.1080/17461391.2012.725103.

Winter, D. (2005). Biomechanics and motor control of human movement (3rd ed.). New York: John Wiley \& Sons. 